\title{
Somatosensory Demands Modulate Muscular Beta Oscillations, Independent of Motor Demands
}

\author{
Freek van Ede and Eric Maris \\ Radboud University Nijmegen, Donders Institute for Brain, Cognition and Behaviour, $6500 \mathrm{HE}$, Nijmegen, The Netherlands
}

Neural oscillations in the beta band $(15-30 \mathrm{~Hz})$ occur coherently throughout the primate somatomotor network, comprising somatomotor cortices, basal ganglia, thalamus, cerebellum, and spinal cord, with the latter resulting in beta oscillations in muscular activity. In accordance with the anatomy of this network, these oscillations have traditionally been associated strictly with motor function. Here we show in humans that somatosensory demands, both in anticipation and during the processing of tactile stimuli, also modulate beta oscillations throughout this network. Specifically, somatosensory demands suppress the degree to which not only cortical activity but also muscular activity oscillates in the beta band. This suppression of muscular beta oscillations by perceptual demands is specific to demands in the somatosensory modality and occurs independent of movement preparation and execution: it occurs even when no movement is required at all. This places touch perception as an important computation within this widely distributed somatomotor beta network and suggests that, at least in healthy subjects, somatosensation and action should not be considered as separable processes, not even at the level of the muscles.

\section{Introduction}

Neural oscillations in the beta band $(15-30 \mathrm{~Hz})$ are a defining feature of one of the most prominent networks in the primate nervous system: the somatomotor network. These oscillations occur coherently throughout this network comprising the primary somatosensory (S1) and motor (M1) cortices (Brovelli et al., 2004; Witham and Baker, 2007), premotor cortex (Ohara et al., 2001), basal ganglia (for review, see Jenkinson and Brown, 2011), thalamus (Marsden et al., 2000; Paradiso et al., 2004), cerebellum (Aumann and Fetz, 2004; Soteropoulos and Baker, 2006), and even the spinal cord (as evidenced from oscillations in muscular activity; Kilner et al., 2004, Baker, 2007).

In agreement with the anatomy of this network, this type of neural activity has traditionally been considered in relation to motor functions such as the preparation and execution of movement (when beta oscillations are suppressed; Pfurtscheller and Lopes da Silva, 1999; Paradiso et al., 2004), holding of posture (when beta oscillations are pronounced; Gilbertson et al., 2005), and the integration of proprioception with action plans during movement (Baker, 2007). At the same time, evidence has accumulated that beta oscillations recorded over the contralateral primary somatomotor cortex are also related to touch perception. Most prominently, several groups have shown that the amplitude of these oscillations is related to tactile perception, with lower prestimulus (Jones et al., 2010; van Ede et al., 2012a) and poststimulus

\footnotetext{
Received Nov. 30, 2012; revised April 5, 2013; accepted April 25, 2013.

Author contributions: F.v.E. and E.M. designed research; F.v.E. performed research; F.v.E. analyzed data; F.v.E. and E.M. wrote the paper.

We gratefully acknowledge the support of the BrainGain Smart Mix Programme of the Ministry of Economic Affairs and the Ministry of Education, Culture and Science of the Netherlands. We also thank our Reviewers for helpful comments and suggestions on earlier versions of the manuscript.

Correspondence should be addressed to Eric Maris, Donders Institute for Brain, Cognition and Behaviour, Radboud University Nijmegen, Montessorilaan 3,6525 HR, Nijmegen, The Netherlands. E-mail: e.maris@donders.ru.nl. DOI:10.1523/JNEUROSCI.5629-12.2013

Copyright $\odot 2013$ the authors $\quad 0270-6474 / 13 / 3310849-10 \$ 15.00 / 0$
}

(Palva et al., 2005) amplitudes being associated with higher detection performance. Moreover, and in accordance with this relation, these oscillations are suppressed over the contralateral primary somatomotor cortex by somatosensory demands, which occurs both in anticipation (Jones et al., 2010; van Ede et al., 2011; Bauer et al., 2012) and during the processing of tactile stimuli (Chatrian et al., 1959; Cheyne et al., 2003; van Ede et al., 2011).

An important question pertains to whether these beta oscillations that are relevant for tactile perception are actually part of the large-scale coherent somatomotor network that has traditionally been studied only in the context of motor tasks. Based on the interareal coupling of these oscillations (Baker et al., 1997; Brovelli et al., 2004), we hypothesize that if this is the case, then beta modulations associated with somatosensory demands should also be observed in motor parts of the network.

To address this, we investigated beta oscillations in muscular activity while human participants performed cued somatosensory identification and detection tasks. In these tasks, cortical beta oscillations are suppressed both in anticipation of and during the processing of tactile stimuli (van Ede et al., 2011, 2012a). We here report highly similar modulations of beta oscillations in muscular activity, even when no movement is required at all. This confirms that the beta oscillations that are modulated by somatosensory demands are part of the larger somatomotor network, placing touch perception as an important computation within this network. In addition, these data suggest that neural activity in populations involved in somatosensation do not occur independently from those involved in action, not even at the level of the muscles.

\section{Materials and Methods}

We analyzed electromyographic (EMG) activity from three experiments in which participants anticipated left- or right-hand tactile stimuli while magnetoencephalography (MEG) and EMG were recorded. The MEG results of these experiments have previously been reported, together with detailed descriptions of the employed materials and methods (Experi- 
ment 1: van Ede et al., 2011; Experiment 2: van Ede et al., 2012b; Experiment 3: van Ede et al., 2012a). We here reiterate those elements that are essential for understanding the results presented in this article. Next to these three experiments, we ran a fourth control experiment in which we only recorded EMG (see Follow-up control experiment).

Participants. Twenty, 19, and 14 healthy subjects (27 males; age range 19-49 years) participated in the three experiments, respectively. Four participants were excluded due to chance-level performance. Experiments were conducted in accordance with guidelines of the local ethics committee (Committee on Research Involving Human Subjects, Region Arnhem-Nijmegen, The Netherlands).

Paradigm. Our main analysis involved the data of Experiment 1 because this experiment contained the largest set of data (both in terms of number of subjects and usable trials) and employed the cleanest stimulation protocol (a single suprathreshold tap to all fingertips of a single hand). In Experiment 1, subjects performed a cued somatosensory identification task. An auditory cue instructed subjects to orient their attention to their left or right hand. At the cued hand, a tactile target would occur 1,2, or $3 \mathrm{~s}$ later. The cue was presented binaurally with the pitch of the cue $(500 / 1000 \mathrm{~Hz})$, indicating the side of the upcoming tactile stimulus. Moreover, the association between pitch and stimulus side was counterbalanced across participants. Because our main analyses focused on lateralized modulations relative to (anticipated) target side, our results can be explained neither by sensory processing of the cue nor by its potentially arousing effect. The tactile target consisted of a stimulation of all fingertips of the cued hand at either proximal or distal sites. We used Braille cells for tactile stimulation (for details, see van Ede et al., 2011). Subjects indicated whether the stimulus was perceived as proximal or distal by pressing a button with the left or right thumb, respectively. On average, subjects responded $1008 \pm 349 \mathrm{~ms}$ after the stimulus.

Crucially, somatosensory demands were independent of response demands because left- and right-hand button presses were equally often required for targets on either hand. This is because the response side depended on the perceived pattern of the stimulus, not its side. Because both patterns (proximal and distal) occurred equally often on either hand, the stimulus side was independent of the required response side. Note that this also holds for the cued side, because the cue predicted the side of the stimulus, not its pattern. Importantly, we also observed this independence in our behavioral data: on average, side-congruent responses (same stimulus and response sides) occurred neither more frequently (proportion of congruent responses: $0.49 \pm 0.01$, denoting mean $\pm 1 \mathrm{SEM} ; t_{(19)}=-1.02 ; p=0.322$ ) nor faster (congruent: $1079 \pm$ 71 , incongruent: $\left.983 \pm 64 \mathrm{~ms} ; t_{(19)}=1.65 ; p=0.116\right)$ than sideincongruent responses. We did note significant biases for some subjects toward either congruent or incongruent responses (as assessed by subject-specific $\chi^{2}$ tests). However, these biases occurred in both directions: some subjects were biased toward congruent responses, while others were biased toward incongruent responses, and hence we did not observe any bias on average. Moreover, these subject-specific biases did not predict the effect of interest, the muscular beta-band modulation. More precisely, neither the subject-specific proportion of congruent responses nor the subject-specific $\chi^{2}$ statistic correlated with the size of this effect (in both cases, $r<0.25, p>0.3$ ).

This spatial independence between stimulus and response sides implies that EMG activity that lateralized according to (anticipated) stimulus side (see Fig. 2) cannot be due to response preparation or execution. We thus deliberately focused our analysis on lateralized EMG activity. In addition to this important aspect of our data, we also (1) directly investigated the effect of any potential response bias (by separating trials by their relation between stimulus and response sides) and (2) ran a control experiment in which we removed the motor response from the trial (see Follow-up control experiment).

EMG recording and analysis. Bipolar surface EMG was recorded from the flexors of the forearms by means of two pairs of $\mathrm{Ag} / \mathrm{AgCl}$ electrodes. Both pairs were placed on the insides of the forearms. Per pair, one electrode was placed $\sim 5 \mathrm{~cm}$ proximal to the wrist and another was placed $\sim 5 \mathrm{~cm}$ distal to the inner tendon of the elbow. This resulted in an electrode placement with an interelectrode distance of $\sim 20 \mathrm{~cm}$. This interelectrode distance is much larger than the distances that are typically used to measure digit-specific EMG. In fact, this electrode placement was chosen because we were interested in the digit nonspecific EMG. In our experiments, investigating covert somatosensory attention, we were interested in such a global measure because the movement of any of the fingers would be considered a motor confound.

Data were analyzed using FieldTrip (Oostenveld et al., 2011). All analyses were done on mean-corrected EMG.

First, we analyzed the frequency content of the EMG traces, as well as their coherence with the cortical activity recorded with MEG (Fig. 1). For this, we divided our dataset into consecutive epochs of $2 \mathrm{~s}$ (regardless of experimental conditions) and applied conventional Fourier analysis in combination with multitapering. Multitapering allows control over spectral smoothing (Percival and Walden, 1993), and for these analyses we applied $4 \mathrm{~Hz}$ smoothing. For the analysis of corticomuscular coherence (i.e., coherence between MEG and EMG; Fig. 1C), we used previously selected MEG channels above left and right primary somatomotor cortices (van Ede et al., 2011). MEG channels above left and right sensorimotor cortices were selected based on stimulusinduced responses to left and right tactile stimuli. Specifically, we contrasted left and right stimuli (right-left) with respect to beta-band amplitude between 100 and $300 \mathrm{~ms}$ poststimulus, and selected the 15 channels with the lowest (highest) value, which were found on the left (right) somatomotor cortex; see also van Ede et al., 2011).

Second, we investigated lateralized modulations in EMG activity as a function of (anticipated) stimulus and response sides (Figs. 2, 3, 4). We contrasted trials in which the (anticipated) stimulus or response side was ipsilateral to the arm from which the EMG was recorded with those trials in which this event occurred contralateral to the recorded EMG. We expressed this contrast as a percentage change: [((ipsilateral - contralateral $) /$ contralateral $) \times 100]$. We did this separately for left and right EMG, and pooled the percentages. (Note that these contrasts are analogous to the previously reported cortical modulations, which were calculated by contrasting activity in MEG channels above left and right primary somatomotor cortices between contralateral and ipsilateral anticipation and stimulation; van Ede et al., 2011). We calculated this contrast for two measures: (1) time- and frequency-resolved power; and (2) high-passfiltered (40 Hz cutoff) and subsequently rectified EMG activity (as an index of muscle tone/contraction). Time-resolved oscillatory power (measure 1) was estimated using a sliding time window of $300 \mathrm{~ms}$ that was advanced in steps of $25 \mathrm{~ms}$ (analogous to the analysis of the cortical MEG data). Statistical significance was evaluated for beta $(15-30 \mathrm{~Hz})$ power lateralization by means of a cluster-based permutation test (Maris and Oostenveld, 2007) in which temporally adjacent thresholded data points were clustered and evaluated under a single permutation distribution (thereby controlling the false-positive rate).

Third, we calculated trial-by-trial correlations between cortical and muscular beta lateralization (Fig. 5). We focused on the anticipatory modulations over the $400-1000 \mathrm{~ms}$ window after the cue, because the anticipatory modulation is most pronounced in this time window (Fig. 2A). Per trial, we calculated the normalized difference between the left $(\mathrm{L})$ and right $(\mathrm{R})$ recording sites: for cortical lateralization, we calculated $[(\mathrm{R}-\mathrm{L}) /(\mathrm{R}+\mathrm{L})]$ and for muscular lateralization we calculated $[(\mathrm{L}-\mathrm{R}) /(\mathrm{L}+\mathrm{R})]$. Separately for left- and right-cued trials, we sorted the trials according to cortical lateralization and placed them into five consecutive bins. For each bin, we then calculated both the average cortical and the average muscular lateralization. Binned data were then averaged across subjects.

Fourth, we calculated the Pearson correlation across subjects between, on the one hand, the muscular lateralization measure, and on the other hand, the cortical lateralization measure and the corticomuscular coherence (Fig. 6). Similarly, we calculated the correlation between, on the one hand, the level of background muscle tone, and on the other hand, the cortical and muscular lateralization measures (Fig. 7C,D). As a measure of background muscle tone, we averaged amplitudes across all frequencies of the calculated power spectra (see step 1 above). For the two correlations with muscle tone, we also evaluated their difference by means of the Hotelling-Williams test for the difference between two dependent correlations (Williams, 1959). For these correlation analyses, we included data from all three experiments. We again focused on the lateralization measures calculated over the last $600 \mathrm{~ms}$ before the anticipated stimulus (i.e., 400-1000 ms postcue in Experiments 1 and 2; 900-1500 
ms postcue in Experiment 3). Note that in Experiment 2 we had presented stimuli at variable cue-target intervals between 0 and $1000 \mathrm{~ms}$. Despite this, we were able to obtain a stimulus-uncontaminated measure of the anticipatory modulation. To achieve this, we calculated timeresolved beta power between 0 and $1000 \mathrm{~ms}$ postcue, using a sliding time window of $300 \mathrm{~ms}$. For every trial, we only used estimates from time bins that occurred before the stimulus. For example, if the stimulus occurred at $800 \mathrm{~ms}$ postcue, the last time bin from which we used the data was between 500 and $800 \mathrm{~ms}$, and data from later time bins were omitted. For every time bin, we first averaged data across trials, and then also across the time bins that showed anticipatory activity (i.e., the bins from $400 \mathrm{~ms}$ postcue onward). Because the time bins never included the stimulus, this resulted in a stimulus-uncontaminated measure of the anticipatory activity. We could estimate anticipatory activity up to $1000 \mathrm{~ms}$, because we had also included catch trials in which no stimuli followed the cue. Because these lateralization measures (muscular: ipsilateral - contralateral; cortical: contralateral - ipsilateral) have negative values (due to a stronger ipsilateralmuscular and contralateral-cortical suppression), we inverted the contrasted values to express the modulation strengths as a positive number.

Differences between experiments. There are two important differences among the three experiments that are considered here. The first difference concerns stimulation protocols. In Experiments 1 and 2, mechanical tactile stimuli were applied to all fingers of one hand, while in Experiment 3 threshold electrical stimuli were applied to the thumb. For this reason, we did not compare stimulus-induced beta modulations across experiments. The second important difference concerns bodily postures. In Experiment 1, participants lay in the MEG (which was put into supine position) with their arms stretched out to grasp our Braille stimulators (for a depiction of one such stimulator, see van Ede et al., 2011, their Supplementary Fig. 1). In Experiment 2, the same Braille stimulators were used, but a subset of participants was recorded while seated. Finally, in Experiment 3, subjects sat while resting their arms on the MEG chair's arm rests, and stimulation electrodes were taped to the thumbs. These different body postures are important when considering the reported differences in background muscle tone (Fig. 7).

Follow-up control experiment. In a follow-up experiment, we addressed two remaining questions. First, we investigated whether the observed EMG modulation by sensory demands is specific to demands in the somatosensory modality. Second, we investigated whether this modulation also occurs when no motor response is required at the end of a trial. To this end, we asked seven subjects (five males; age range, 27-51 years) who previously showed this modulation, to return to the laboratory for a follow-up experiment. In this experiment, we only recorded EMG and used the same electrode placement as described above.

The experiment consisted of two cued detection tasks, one in the somatosensory modality and one in the visual modality. In both tasks, we presented auditory cues (the same cues that were used in Experiment 1) that instructed subjects to orient their attention to either the left or the right side (in the case of the tactile task, the left or right hand; in the case of the visual task, the left or right side of the screen). One second after this cue, a stimulus occurred with probability 0.5 at the cued location, and the subjects' task was to judge whether or not they felt (tactile blocks) or saw (visual blocks) a stimulus. Tactile stimuli were applied for $1 \mathrm{~ms}$ to the left or right median nerve using either of two constant-current stimulators (type DS7A, Digitimer) that were taped to the body. Visual stimuli were presented for a single frame $(16.7 \mathrm{~ms})$ on the left or right side of the screen, and consisted of filled white circles with a diameter of $\sim 1$ $\mathrm{cm}$. Before the experiment, intensities of the stimuli (respectively, the electrical current and the visual contrast) were adjusted to a level where the stimuli were just above perceptual threshold (based on verbal report). Tactile and visual blocks (each containing 10 trials) were randomly interleaved. The relevant modality was indicated to the subject by means of the text "tactile task" or "visual task" that remained on the screen throughout the block.

In this control experiment, the trials did not include a motor response. Instead, subjects had to count the number of stimuli presented within a block (which ranged from 0 to 10) and to report this number verbally only at the end of each block. Tasks were equated on difficulty: on average, subjects reported the number of presented stimuli correctly in $56.28 \pm 2.56 \%$ (visual) and $51.75 \pm 3.92 \%$ (tactile) of the blocks. The average absolute deviations from the correct numbers were, respectively, $0.83 \pm 0.15 \%$ and $0.82 \pm 0.13 \%$.

Before running this follow-up experiment, we had observed that the EMG modulation of interest requires the presence of background muscle activity (Fig. 7). To achieve this in our control experiment, we asked subjects to hold up a tube (1000 $\mathrm{cm}$ wide, $7 \mathrm{~cm}$ diameter, $956 \mathrm{~g}$ ) throughout the experiment. Subjects were in seated position, rested their elbows on the chair, and lifted the tube with both hands (palms facing upward). Importantly, this motor engagement does not induce a confound in our results because the tube was held up in the same way regardless of the anticipated side and regardless of the modality of the block (see also Fig. 8D). Our analyses focused on the difference in activity between ipsilateral and contralateral anticipation and the difference between tactile and visual blocks.

\section{Results}

\section{Cortex and muscle couple at beta frequency}

Throughout the cued tactile identification task (see Materials and Methods), muscle activity recorded at the flexors of the forearms showed rhythmic activity in the beta range (15-30 Hz; denoted "muscular beta oscillations"). Figure $1 A$ depicts an example EMG trace showing these oscillations. These oscillations were observed in the majority of our subjects as revealed by the grand average EMG power spectrum (Fig. 1B). Moreover, these oscillations were coherent with those recorded above contralateral primary somatomotor cortex (Fig. 1C,D). These observations are consistent with a large body of literature in which this type of activity has been investigated during steady contraction in the context of motor tasks (Baker et al., 1997; Salenius et al., 1997; Schoffelen et al., 2005).

\section{Muscular beta oscillations are suppressed by strictly somatosensory demands}

We investigated EMG activity that lateralized in anticipation of (Fig. 2A) and during the sensory processing of (Fig. 2B) a tactile stimulus to the left or right hand. In several previous reports, we and others have documented that such lateralized tactile anticipation and stimulus processing involve an accordingly lateralized modulation of cortical alpha and beta oscillations (see introduction). Because of the coupling between cortical and muscular beta oscillations (Fig. 1C,D), it is conceivable that the muscular beta oscillations are similarly modulated. Precisely this can be observed in the time-frequency representations of the difference in power between ipsilateral and contralateral EMG (Fig. 2, top panels) (for the corresponding cortical modulations, see van Ede et al., 2011, their Figs. $2 A$ and Supplementary Fig. 5A). Time-resolved power differences for the beta band (15-30 Hz; middle panels) reveal the robustness of these modulations (shading represents \pm 1 SEM; $p$ values indicate the significance of the temporal clusters; see Materials and Methods). We further assessed whether this lateralization was constituted by an ipsilateral decrease or a contralateral increase by investigating the temporal development of the anticipatory modulation (Fig. 2A, middle) separately for ipsilateral and contralateral EMG. As we observed in cortex (van Ede et al., 2011), the lateralization results from a suppression of beta oscillations in populations representing the task-relevant hand: muscular beta-power ipsilateral to the anticipated stimulus decreases with time after cue $\left(r=-0.69 \pm 0.08 ; t_{(19)}=-8.52, p<\right.$ $0.001)$. See also Figure $8 D$ for the ipsilateral nature of this modulation.

\section{The muscular beta modulation is independent of movement preparation and execution}

Several aspects rule out movement preparation and execution as possible explanations for the observed muscular beta modula- 
tions. First, in our task (anticipated) stimulus and response sides were spatially uncorrelated, both in design and in response behavior (see Materials and Methods). The lateralized modulations with respect to the stimulus side (Fig. 2) can therefore not be attributed to response preparation and execution. We could further show this in our data. For this, we focused on the anticipatory modulation and used the following logic: if this modulation is due to response preparation, then the lateralized pattern observed in Figure $2 A$ should reverse between trials in which the stimulus is followed by a response on the same versus the opposite side. In contrast, we observed a highly similar anticipatory modulation between these two sets of trials (Fig. 3). This directly shows that the lateralization relative to the anticipated stimulus side cannot be attributed to response preparation. Moreover, as we will show later (Fig. 8), this anticipatory modulation also occurs when no response is required at all. In addition to this analysis, we also directly investigated EMG activity that lateralized according to response side (Fig. 4). This pattern of EMG activity looks fundamentally different from the pattern associated with tactile anticipation and stimulus processing: it is of opposite sign, occurs in all frequencies, and is more than an order of magnitude larger.

Finally, our observations can also not be attributed to non-response-related movement during anticipation and processing of the tactile stimuli. This is evidenced by the lack of modulation in muscle tone (bottom panels Fig. 2; compare with the massive increase during actual motor behavior in Fig. 4). Thus, while somatosensory demands reduce the degree to which muscular output oscillates in the beta range, they leave overall muscle output (as indexed by rectified, high-pass-filtered, EMG activity) unaltered. The modulation presented here can therefore not be explained by (preparation of) movement of the fingers during tactile anticipation and stimulus processing. Rather, as we will show below, it likely occurs as a consequence of the cortical, somatosensory-related modulation that propagates through the somatomotor network.

The muscular beta modulation reflects a spillover of the cortical beta modulation onto the muscles

If the muscular beta modulation reflects a spillover of the cortical modulation onto the muscles, then it is expected (1) to correlate with the cortical modulation on a
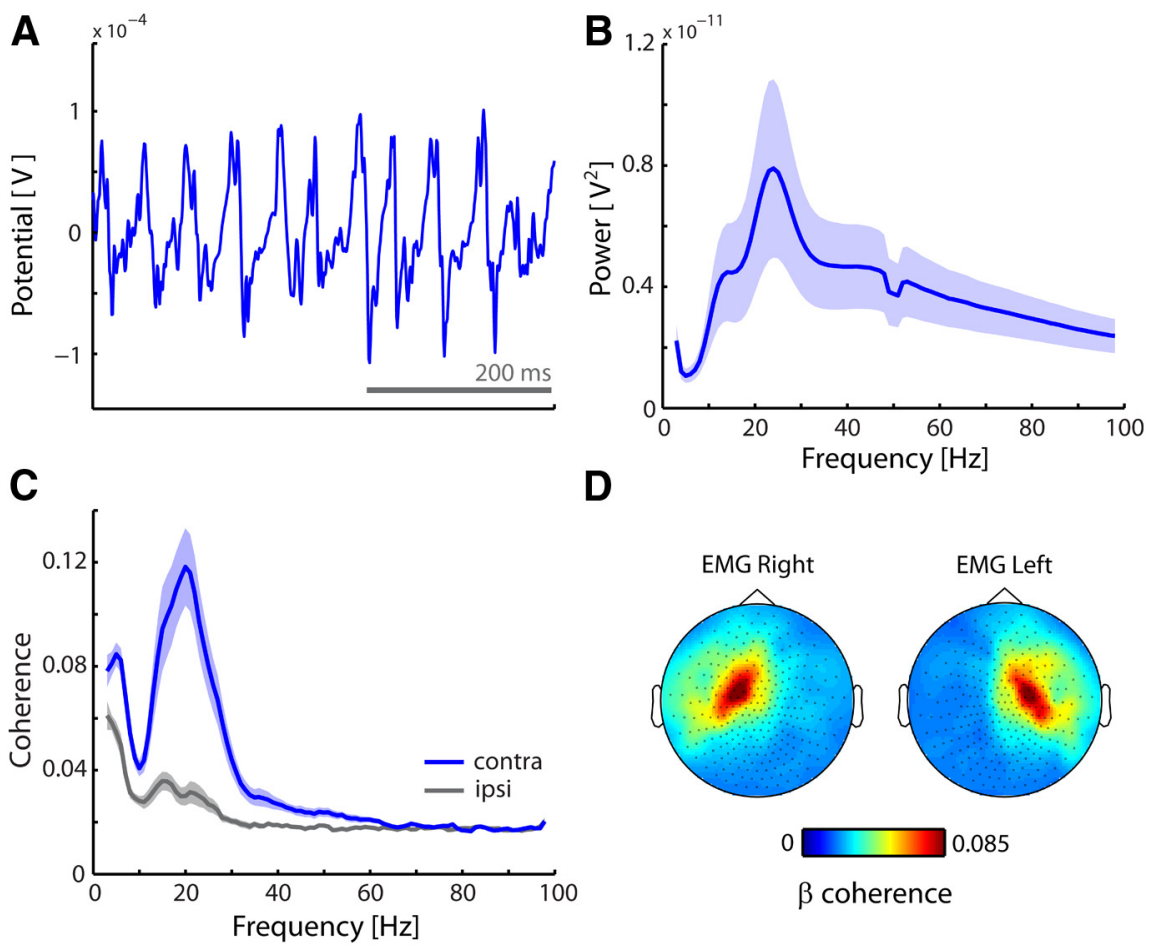

Figure 1. Muscular activity oscillates at beta frequency, coherent with contralateral cortex. $A$, Example mean-corrected EMG trace. $\boldsymbol{B}$, Grand average muscular power spectrum. $\boldsymbol{C}$, Grand average coherence spectra between EMG and MEG channels above contralateral and ipsilateral primary somatomotor cortices. $\boldsymbol{D}$, Topographies of the beta coherence with the left and right forearm EMG. Blue and gray shadings indicate \pm 1 SEM. Data are from Experiment 1 .
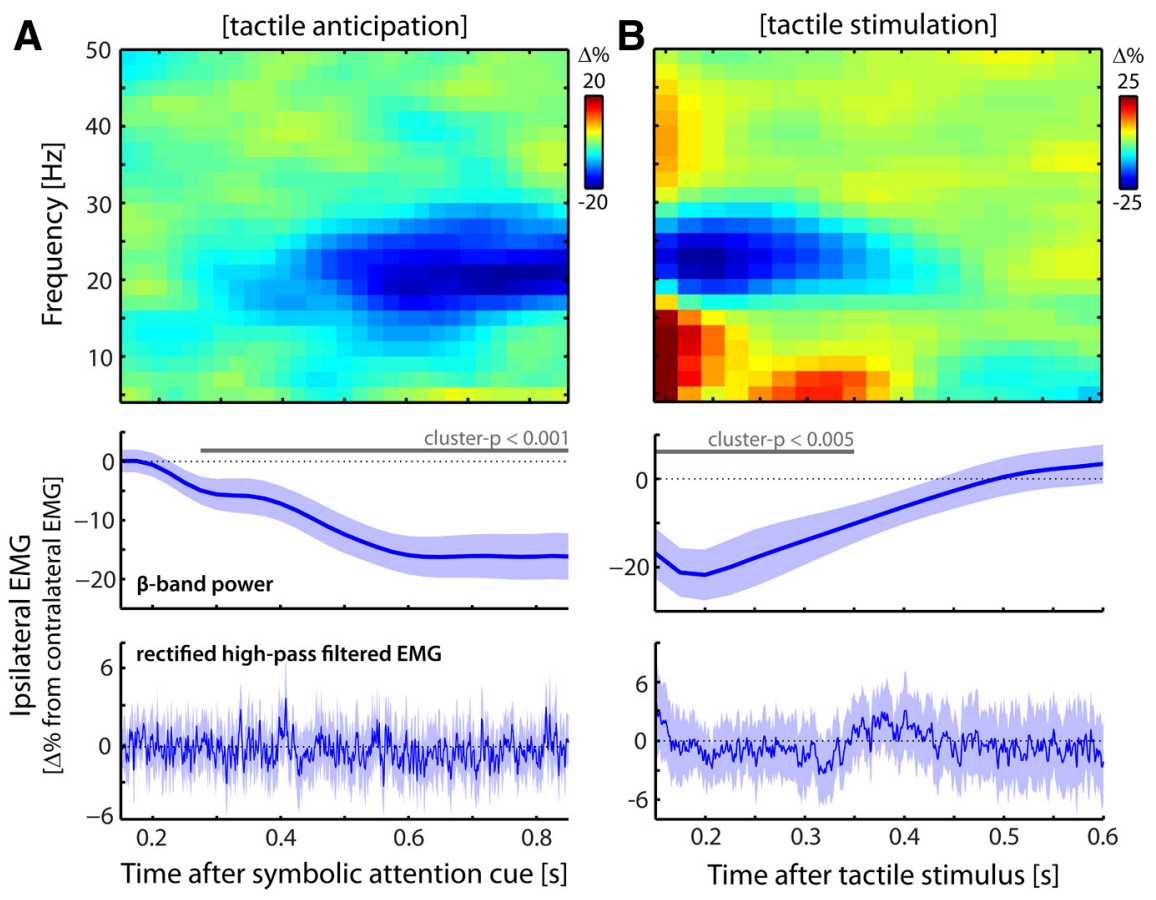

Figure 2. Somatosensory demands modulate muscular beta oscillations. $\boldsymbol{A}, \boldsymbol{B}$, Modulations of muscular beta oscillations by lateralized tactile anticipation $(\boldsymbol{A})$ and tactile stimulation $(\boldsymbol{B})$. Modulations are expressed as the difference between EMG recorded on the hand ipsilateral and contralateral to the (expected) event (ipsilateral - contralateral, expressed as a percentage change). Compare with the contralateral - ipsilateral contrasts in the MEG data from the primary somatomotor cortices (see van Ede et al., 2011, their Figs. 2 A, Supplementary Fig. 5A, respectively). Middle, Time courses of these contrasts for extracted beta-band power. Solid gray lines indicate significant clusters (see Materials and Methods). Dotted gray lines indicate the null hypothesis of no difference between contralateral and ipsilateral EMG. Bottom, Changes in muscle tone (high-pass-filtered and rectified EMG). Blue shadings represent \pm 1 SEM. Data are from Experiment 1 . 

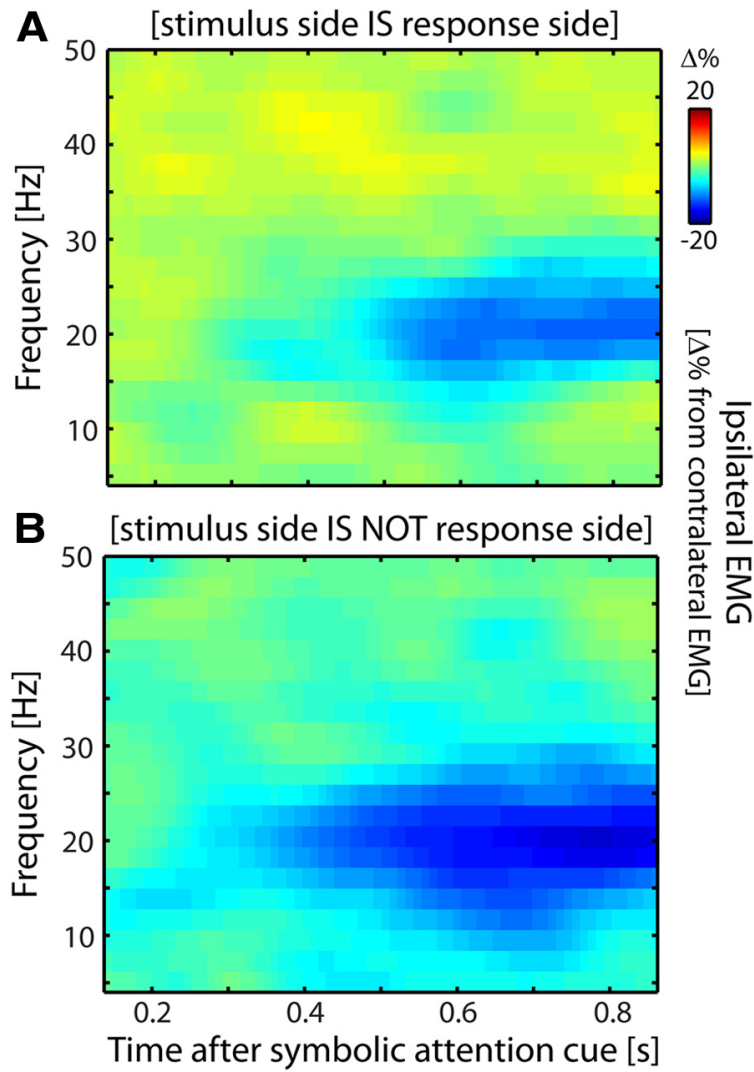

Figure 3. The EMG lateralization occurs regardless of response side. $A, B$, Identical to Figure $2 A$, except that data are split into trials in which the response was made on the same side as the stimulus $(\boldsymbol{A})$ and trials in which the response was made on the opposite side $(\boldsymbol{B})$. Lateralization is depicted relative to the anticipated stimulus side. Data are from Experiment 1.

trial-by-trial basis, (2) to correlate with the strength of the cortical modulation across subjects, and (3) to scale with corticomuscular coherence. We assessed these predictions with regard to the anticipatory modulation (Fig. 2A) (see Materials and Methods). Figure $5 \mathrm{~A}$ shows the trial-by-trial relation between cortical and muscular states (as indexed by beta power lateralization) in anticipation of left and right tactile stimuli (note that we binned data to allow averaging across participants). Clearly, a strong correlation exists between cortical and muscular states. For example, when cortex is in a state of "left anticipation" (i.e., when beta power is lower in right compared with left MEG), so are the muscles (i.e., when beta power is lower in left compared with right EMG). This correlation is highly robust across subjects: for the binned data, this correlation was $r=0.74 \pm 0.07\left(t_{(19)}=\right.$ $10.81, p<0.001)$. Moreover, as predicted, this subject-specific across-trial correlation was stronger for subjects with a higher corticomuscular coherence $(r=0.64 ; p<0.01)$. Furthermore, analogous to the pattern of corticomuscular coherence (Fig. $1 C, D)$, this across-trial correlation between cortical and muscular power was specific to the beta band (Fig. $5 B$ ) and to channels above the contralateral primary sensorimotor cortices (Fig. 5C).

We also assessed the relation between the cortical and muscular modulations across subjects. For this, we combined data from three experiments, all in which lateralized tactile stimuli were anticipated while MEG and EMG were recorded (see Materials and Methods). As depicted in Figure 6, the strength of the muscular modulation correlated with the strengths of both the cortical modulation (Fig. 6A) and the corticomuscular coherence (Fig. 6B). These correlations remained significant, even when
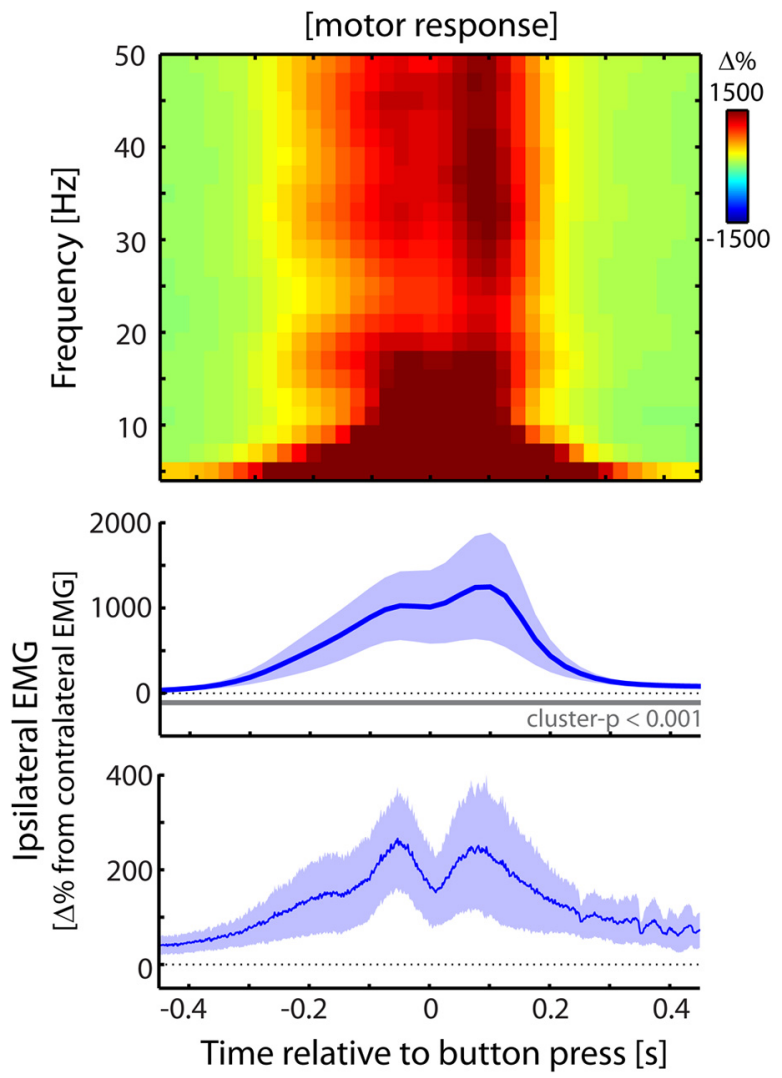

Figure 4. EMG activity related to overt motor behavior looks fundamentally different. Identical to Figure 2, except muscular activity is now contrasted between ipsilateral and contralateral motor responses (i.e., button presses with the left or right thumb). Data are from Experiment 1.

controlling for the factor "experiment" (i.e., when we subtracted the mean values per experiment from all the observations in that experiment before calculating these correlations; $r=0.46, p<$ 0.001 and $r=0.44, p<0.005$, respectively). At the same time, we also noted an interesting difference among the three experiments that we further explore below.

\section{The muscular beta modulation is gated by factors determining background muscle tone}

The three experiments differed drastically in absolute levels of EMG activity (Fig. $7 A ; F_{(2,46)}=4.3, p<0.05$ ), likely due to different postural positions across the experiments (see Materials and Methods). This allowed us to investigate to what extent both the cortical and the muscular modulations depend on background muscle tone. In contrast to the cortical modulation, the muscular beta modulation strongly depends on background muscle tone (Fig. $7 B$ ). In fact, in the experiment in which very little EMG power was observed, we did not observe an anticipatory modulation of muscular beta oscillations $(p>0.1)$, despite a robust cortical modulation. The relation of background muscle tone with the strength of the muscular beta modulation (and likewise, the absence of this relation with the cortical beta modulation) was also revealed by a correlation analysis across subjects (Fig. 7C,D). A significant correlation with background muscle tone was observed only for the muscular modulation $\left(r_{\text {muscle }}=\right.$ $\left.0.448, p=<0.05 ; r_{\text {cortex }}=0.107, p>0.45\right)$. Completely in line with this observation, the correlation with the muscular modulation was significantly larger than the one with the cortical modulation $\left(t_{(46)}=2.4, p<0.05\right)$. Together, these data show that the 
hypothesized propagation from cortex to muscle is gated by factors determining background muscle tone.

\section{The modulation of muscular beta} oscillations by sensory demands is specific to the somatosensory modality and does not require a motor response in the trial

In a follow-up control experiment (see Materials and Methods) we addressed whether the muscular beta modulation by sensory demands is specific to the somatosensory modality. To this end, we recorded EMG activity while subjects anticipated either an upcoming tactile or an upcoming visual stimulus. As before, we calculated the lateralized difference in EMG activity as a function of anticipated stimulus side (left hand, right hand, or visual field, respectively). As is evident from Figure $8 A-C$, the modulation is specific to the somatosensory modality (tactile: $t_{(6)}=-2.89, p<0.05$; visual: $t_{(6)}=$ $-0.11, p>0.9$; tactile minus visual: $t_{(6)}=$ $-3.45, p<0.05)$. Importantly, this difference cannot be explained by differences in overall muscle tone between the visual and tactile tasks: the overall power in the EMG was highly similar $\left(t_{(6)}=-1.30\right.$, $p>0.2)$ between our tactile and visual detection tasks (Fig. 8D). In fact, the only aspect with respect to which the power spectra differ, concerns our effect of interest: a selective attenuation in the beta band, only in EMG recordings ipsilateral to the anticipated stimulus, and only during tactile anticipation (Fig. 8D).

In this control experiment, the subjects did not have to give a motor response at the end of the trial (see Materials and Methods). Crucially, this did not alleviate the modulation of muscular beta oscillations. These data thus further strengthen the notion that the reported modulation of muscular beta oscillations can occur independent of motor demands.

\section{Discussion}

We observed that the previously documented cortical modulation of beta oscillations by somatosensory demands-in anticipation of and during the processing of tactile stimuli-also manifests itself in oscillatory patterns of muscular activity, independent of movement preparation and execution. This implies that these somatosensory-relevant beta oscillations are part of the large-scale somatomotor network, placing touch perception as an important computation in this network. In addition, these data suggest that, despite specific behavioral demands, it might not be possible to engage populations involved in somatosensation and action independently, not even at the level of the muscles.

The observed muscular beta modulation is likely explained by propagation of this modulation along anatomical pathways from primary somatomotor cortex to muscle. For example, this might


Figure 5. Cortical state predicts muscular state on a trial-by-trial basis. $\boldsymbol{A}$, Data points represent normalized lateralization dices that were binned according to cortical lateralization in the last $600 \mathrm{~ms}$ before the anticipated tactile stimulus (400-1000 , the time window in which this modulation is most pronounced; Fig. $2 A)$. This was done for both left-and right-hand for right compared with left primary somatomotor cortices together with lower power for left compared with right EMG (vice versa for neural state "right anticipation"). B, Frequency spectrum of the trial-by-trial correlation between cortical and muscular lateralization. Blue and red lines represent left and right cued trials, respectively. Shadings represent \pm 1 SEM. $\boldsymbol{C}$, Topographies of the trial-by-trial correlation between cortical and muscular beta power, separately for the left and rightEMG. Data are from Experiment 1.

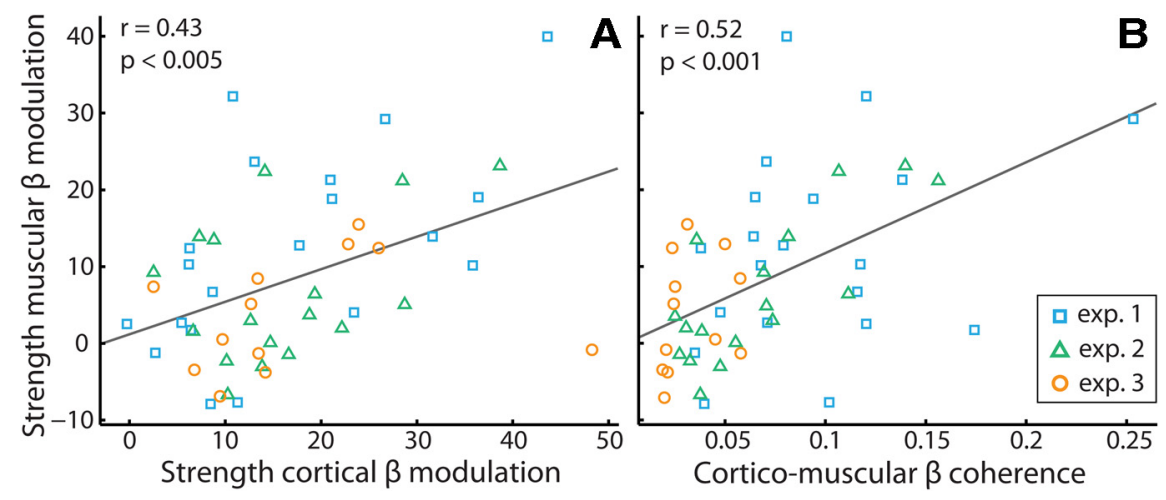

Figure 6. Strength of muscular beta modulation depends on strength of cortical modulation and corticomuscular coupling. $\boldsymbol{A}$, Correlation between strengths (see Materials and Methods) of the cortical and muscular beta modulations in anticipation of a lateralized tactile stimulus (compare with Fig. 2A). Data points represent individual participants. Data are shown from three anticipated stimulus (400-1000 ms postcue for Experiments 1 and 2; $900-1500$ ms postcue for Experiment 3). $\boldsymbol{B}$, Identical to $\boldsymbol{A}$, except corticomuscular coherence is plotted as the predictor.

occur from S1 (where it is required in our task) to M1 (Brovelli et al., 2004) and then via pyramidal tract neurons to alpha-motor neurons in the spinal cord, regulating muscular output. Alternatively, this might also occur through direct efferent pathways that exist between S1 and the spinal cord (Coulter and Jones, 1977; Matyas et al., 2010). Indeed, three observations suggest that the cortical (van Ede et al., 2011) and muscular (present article) modulations represent the same modulation occurring in the distinct populations within the network: the cortical and muscular modulations (1) have highly similar modulation profiles, (2) are both constituted by a suppression within the populations related to the task-relevant hand, and (3) are highly correlated, both across trials and participants. In addition to the cortical requirements of our task, the cortical origin of the beta modulation is also confirmed by the fact that only the muscular modulation depends on muscle tone. In fact, if the muscular modulation would drive the cortical modulation, a similar dependency should be observed in cortex. We thus propose that the 

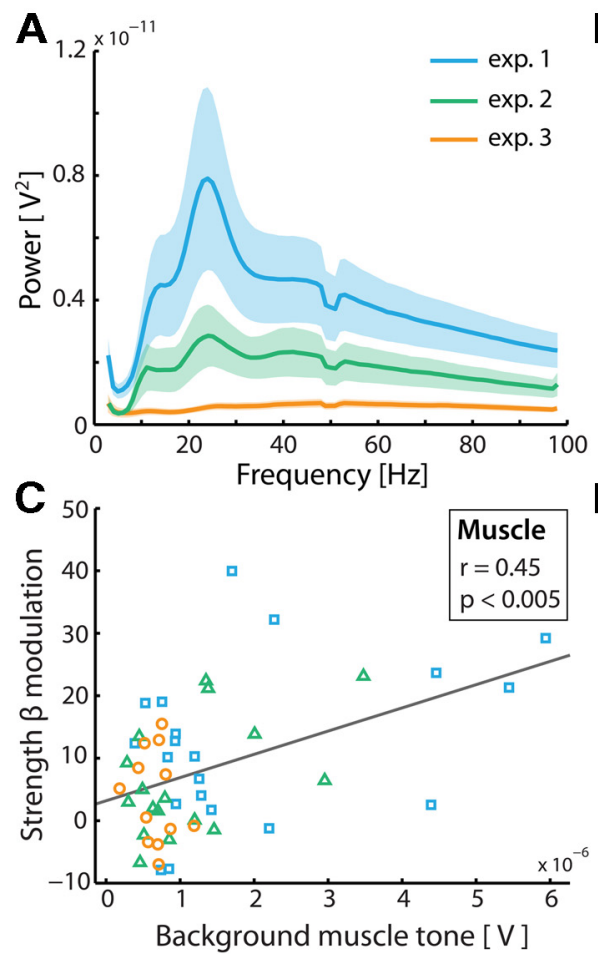

B


Figure 7. Muscular but not cortical beta modulation depends on background muscle tone. $A$, Grand average muscular power spectra for the three experiments. Colored shadings represent \pm 1 SEM. $\boldsymbol{B}$, Strengths of the cortical and muscular beta modulations in anticipation of a tactile stimulus for the three experiments in $\boldsymbol{A}$. Error bars represent \pm 1 SEM. $p$ values represent statistical significance with respect to the null hypothesis of no lateralization (strength $=0$ ). $C$, Correlation between background muscle tone and strength of the anticipatory muscular beta modulation. Background muscle tone is indexed by the average amplitude across all frequencies of the calculated background EMG power spectra (compare with $\boldsymbol{A}$; see Materials and Methods for details). $\boldsymbol{D}$, Similar to $C$, except the cortical modulation is plotted as the criterion variable.

muscular beta modulation reflects a spillover from cortex to muscles along descending anatomical pathways. Considering this hypothesis, two points must be clarified. First, the somatosensory nature of our task does not necessarily imply that the modulation is also initiated in S1. In fact, it might also be initiated elsewhere in the network and propagate to S1 to assist tactile processing. Second, in contrast to this propagation profile for the modulation of beta oscillations, their generation might involve a more complex interplay between descending and ascending corticospinal pathways (Baker, 2007).

A critical feature of the muscular beta modulation reported here is the absence of a concurrent change in muscle tone (i.e., muscle contraction). However, this observation by itself does not preclude a motor account of our data. For example, there is ample evidence (Pfurtscheller and Neuper, 1997; Miller et al., 2010) that processes such as motor preparation, motor imagery, and motor intent (all processes without motor output) also modulate cortical beta oscillations. (To our knowledge, this has not yet been established at the muscular level.) Crucially, we also argue that these processes cannot account for our observations. Considering movement preparation, the muscular modulation occurred regardless of movement side (Fig. 3) and occurred even when no response was required at all (Fig. 8). Considering imagery or intent, it is unlikely that participants imagined or intended moving their hand, as this would not help them in the perceptual tasks. This is most obvious in our control experiment, in which the tactile stimulator was taped to the body (Fig. 8). We thus conclude that the muscular modulations reported here must be due strictly to somatosensory demands.
The main implication of our observations is that the somatosensory-related modulation of beta oscillations (and therefore touch perception) must be understood in relation to the large-scale somatomotor beta network. Previous studies already pointed at the interplay between beta oscillations in this network and somatosensation. On the one hand, studies showed that somatosensation might be critical to the occurrence of beta oscillations in this network: (1) S1 drives M1 beta oscillations (in a Granger causality sense; Brovelli et al., 2004); and (2) coherence of muscular (Fisher et al., 2002; Kilner et al., 2004) and corticomuscular (Riddle and Baker, 2005) beta oscillations is altered by alterations in afferent (periphery to cortex) sensory processing. On the other hand, studies suggested that beta oscillations in this network might also contribute to somatosensory perception. For example, the timing of action potentials of cells in S1 (including cutaneous areas $3 \mathrm{~b}$ and 1) can be influenced by beta oscillations recorded elsewhere in the network (M1, as shown by Witham et al., 2007). Along the same line, recent MEG studies showed that the amplitude of beta oscillations above contralateral primary somatomotor cortex is inversely related to touch perception (Palva et al., 2005; Jones et al., 2010; van Ede et al., 2012a,b), and is accordingly suppressed in anticipation of and during the processing of tactile stimuli (Jones et al., 2010; van Ede et al., 2010, 2011; Bauer et al., 2012). An important insight from the current work is that these somatosensory-relevant beta oscillations are in fact part of the largescale somatomotor beta network. This underscores the notion that touch perception is an important computation within this network, even when no movement is required. At this point, it is important to clarify that we do not want to claim that the muscular beta modulation is itself functionally relevant for tactile perception (i.e., that the muscle is doing somatosensory processing). Rather, our data provide evidence for the notion that this type of signal (beta oscillations in a large-scale coherent network), must be understood not only in relation to motor computations, but also somatosensory computations (see also Lalo et al., 2007). While previous studies had already implicated beta oscillations in tactile perception (Jones et al., 2010; van Ede et al., 2010, 2011, 2012a,b), we here show that these oscillations are in fact the same as those often studied in the context of motor tasks (i.e., the beta oscillations that are visible in the EMG).

Strikingly, our data suggest that task-related modulations of neural activity do not occur independently in neural populations involved in somatosensation and action, not even when behavioral demands require their independence, as in our task. This is likely due to the fact that distinct populations in the somatomotor network are strongly interconnected. This strong interconnectedness most likely results from the high frequency with which they interact. In fact, during movement, populations involved in somatosensation and action are in a continuous dialogue: action causes somatosensation, leading to, for example, adjusted action. However, in several situations (such as in our experiments), touch perception is independent from action. The 
observation reported here that in such situations muscular activity is nevertheless modulated therefore reveals a constraint on the flexibility with which neural populations involved in somatosensation and action can independently modulate their activity. Put differently, our data show that, from a physiological perspective, somatosensation and action should not be considered as two fully separable processes. This conclusion is further supported by recent rodent studies implicating primary motor cortex in tactile perception (Ferezou et al., 2007) and primary somatosensory cortex in motor control (Matyas et al., 2010). The current work demonstrates this inherent dependence between somatosensation and action in humans. In future investigations, it would be interesting to address whether this dependence persists in patients who have lost muscle control after brain or spinal cord injury.

In cortex, somatosensory demands modulate not only beta but also alpha oscillations (van Ede et al., 2011). Moreover, in cortex these oscillations are strongly coupled: they show highly similar modulation profiles (at least during tactile anticipation), and their spontaneous fluctuations are highly correlated (van Ede et al., 2012a). In contrast, the associated muscular modulations are restricted to the beta band. This suggests that, in primary somatomotor cortex, factors that modulate alpha oscillations also modulate beta oscillations (and vice versa). At the same time, oscillations in these two bands propagate differently throughout the somatomotor network, with only beta oscillations affecting spinal populations (note that these data do not imply that alpha oscillations do not play a role in muscular function; rather, they show that, concerning the somatosensory modulation of these oscillations, only the modulation in the beta band propagates to the muscles). As a result, spinal beta oscillations (visible in the EMG) might be modulated by beta modulations elsewhere in the network, a phenomenon of which the present work showcases one example.

Finally, our results have a practical implication by showing that, given an appropriate posture, beta oscillations in muscular activity can index the state of neural activity as it occurs elsewhere in the somatomotor network. Compared with MEG and EEG, this allows for an easier, cheaper, more mobile, and spatially more specific way of assessing the state of the somatomotor network during various tasks, both in fundamental as well as clinical research settings.

We conclude that touch perception is an important computation within the widely distributed somatomotor beta network, and that somatosensation and action should not be considered as separable processes, not even at the level of the muscles.

\section{References}

Aumann TD, Fetz EE (2004) Oscillatory activity in forelimb muscles of behaving monkeys evoked by microstimulation in the cerebellar nuclei. Neurosci Lett 361:106-110. CrossRef Medline

Baker SN (2007) Oscillatory interactions between sensorimotor cortex and the periphery. Curr Opin Neurobiol 17:649-655. CrossRef Medline tactile anticipation]
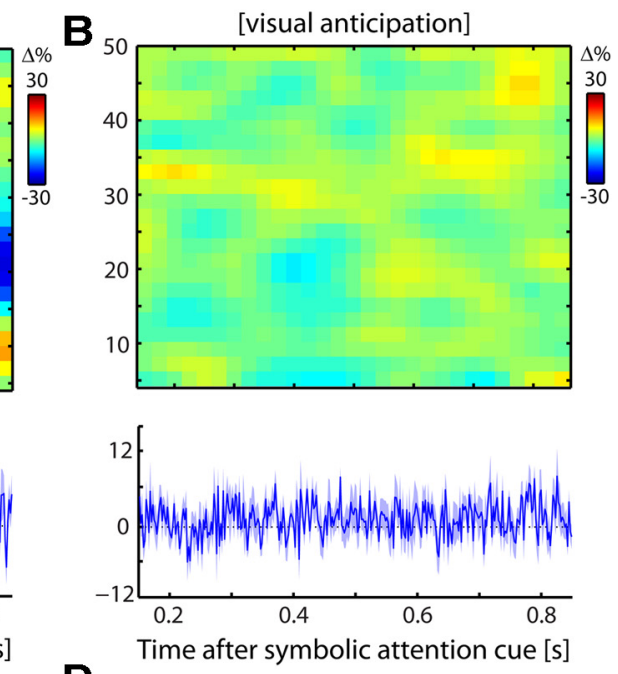

$\mathbf{D}$

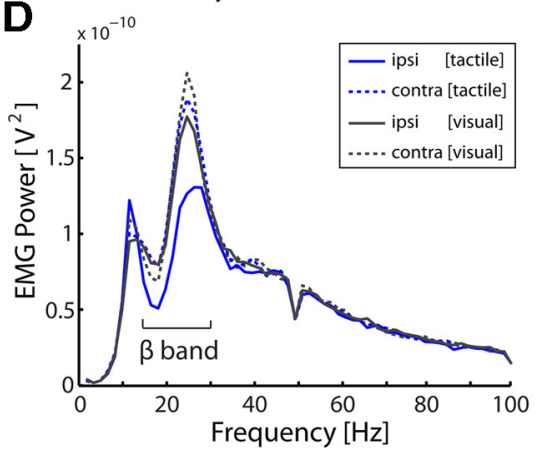

Figure 8. The modulation of muscular beta oscillations by sensory demands is specific to the somatosensory modality and does not require a motor response in the trial. $\boldsymbol{A}, \boldsymbol{B}$, Modulations of muscular oscillations by lateralized tactile $(\boldsymbol{A})$ and visual $(\boldsymbol{B})$ cipation. Conventions are as in Figure 2A. Data are from our follow-up experiment (see Materials and Methods), in which stimuli. Because these power spectra were calculated over the 400-1000 ms postcue window, they also reveal the anticipatory modulation of interest (see the attenuation in $15-30 \mathrm{~Hz}$ power in the ipsilateral EMG during tactile anticipation).

Baker SN, Olivier E, Lemon RN (1997) Coherent oscillations in monkey motor cortex and hand muscle EMG show task-dependent modulation. J Physiol 501:225-241. CrossRef Medline

Bauer M, Kennett S, Driver J (2012) Attentional selection of location and modality in vision and touch modulates low-frequency activity in associated sensory cortices. J Neurophysiol 107:2342-2351. CrossRef Medline

Brovelli A, Ding M, Ledberg A, Chen Y, Nakamura R, Bressler SL (2004) Beta oscillations in a large-scale sensorimotor cortical network: directional influences revealed by Granger causality. Proc Natl Acad Sci U S A 101:9849-9854. CrossRef Medline

Chatrian GE, Petersen MC, Lazarte JA (1959) The blocking of the rolandic wicket rhythm and some central changes related to movement. Electroencephalogr Clin Neurophysiol 11:497-510. CrossRef Medline

Cheyne D, Gaetz W, Garnero L, Lachaux JP, Ducorps A, Schwartz D, Varela FJ (2003) Neuromagnetic imaging of cortical oscillations accompanying tactile stimulation. Brain Res Cogn Brain Res 17:599-611. CrossRef Medline

Coulter JD, Jones EG (1977) Differential distribution of corticospinal projections from individual cytoarchitectonic fields in the monkey. Brain Res 129:335-340. CrossRef Medline

Ferezou I, Haiss F, Gentet LJ, Aronoff R, Weber B, Petersen CC (2007) Spatiotemporal dynamics of cortical sensorimotor integration in behaving mice. Neuron 56:907-923. CrossRef Medline

Fisher RJ, Galea MP, Brown P, Lemon RN (2002) Digital nerve anaesthesia decreases EMG-EMG coherence in a human precision grip task. Exp Brain Res 145:207-214. CrossRef Medline

Gilbertson T, Lalo E, Doyle L, Di Lazzaro V, Cioni B, Brown P (2005) Existing motor state is favored at the expense of new movement during 
13-35 Hz oscillatory synchrony in the human corticospinal system. J Neurosci 25:7771-7779. CrossRef Medline

Jenkinson N, Brown P (2011) New insights into the relationship between dopamine, beta oscillations and motor function. Trends Neurosci 34: 611-618. CrossRef Medline

Jones SR, Kerr CE, Wan Q, Pritchett DL, Hämäläinen M, Moore CI (2010) Cued spatial attention drives functionally relevant modulation of the mu rhythm in primary somatosensory cortex. J Neurosci 30:13760-13765. CrossRef Medline

Kilner JM, Fisher RJ, Lemon RN (2004) Coupling of oscillatory activity between muscles is strikingly reduced in a deafferented subject compared with normal controls. J Neurophysiol 92:790-796. CrossRef Medline

Lalo E, Gilbertson T, Doyle L, Di Lazzaro V, Cioni B, Brown P (2007) Phasic increases in cortical beta activity are associated with alterations in sensory processing in the human. Exp Brain Res 177:137-145. CrossRef Medline

Maris E, Oostenveld R (2007) Nonparametric statistical testing of EEG- and MEG-data. J Neurosci Methods 164:177-190. CrossRef Medline

Marsden JF, Ashby P, Limousin-Dowsey P, Rothwell JC, Brown P (2000) Coherence between cerebellar thalamus, cortex and muscle in man: cerebellar thalamus interactions. Brain 123:1459-1470. CrossRef Medline

Matyas F, Sreenivasan V, Marbach F, Wacongne C, Barsy B, Mateo C, Aronoff R, Petersen CC (2010) Motor control by sensory cortex. Science 330: 1240-1243. CrossRef Medline

Miller KJ, Schalk G, Fetz EE, den Nijs M, Ojemann JG, Rao RP (2010) Cortical activity during motor execution, motor imagery, and imagery-based online feedback. Proc Natl Acad Sci U S A 107:4430-4435. CrossRef Medline

Ohara S, Mima T, Baba K, Ikeda A, Kunieda T, Matsumoto R, Yamamoto J, Matsuhashi M, Nagamine T, Hirasawa K, Hori T, Mihara T, Hashimoto N, Salenius S, Shibasaki H (2001) Increased synchronization of cortical oscillatory activities between human supplementary motor and primary sensorimotor areas during voluntary movements. J Neurosci 21:9377-9386. Medline

Oostenveld R, Fries P, Maris E, Schoffelen JM (2011) FieldTrip: open source software for advanced analysis of MEG, EEG, and invasive electrophysiological data. Comput Intell Neurosci 2011:156869. CrossRef Medline

Palva S, Linkenkaer-Hansen K, Näätaänen R, Palva JM (2005) Early neural correlates of conscious somatosensory perception. J Neurosci 25:5248-5258. CrossRef Medline

Paradiso G, Cunic D, Saint-Cyr JA, Hoque T, Lozano AM, Lang AE, Chen R (2004) Involvement of human thalamus in the preparation of self-paced movement. Brain 127:2717-2731. CrossRef Medline
Percival DB, Walden AT (1993) Spectral analysis for physical applications: multitaper and conventional univariate techniques. Cambridge, UK: Cambridge UP.

Pfurtscheller G, Lopes da Silva FH (1999) Event-related EEG/MEG synchronization and desynchronization: basic principles. Clin Neurophysiol 110:1842-1857. CrossRef Medline

Pfurtscheller G, Neuper C (1997) Motor imagery activates primary sensorimotor area in humans. Neurosci Lett 239:65-68. CrossRef Medline

Riddle CN, Baker SN (2005) Manipulation of peripheral neural feedback loops alters human corticomuscular coherence. J Physiol 566:625-639. CrossRef Medline

Salenius S, Portin K, Kajola M, Salmelin R, Hari R (1997) Cortical control of human motoneuron firing during isometric contraction. J Neurophysiol 77:3401-3405. Medline

Schoffelen JM, Oostenveld R, Fries P (2005) Neuronal coherence as a mechanism of effective corticospinal interaction. Science 308:111-113. CrossRef Medline

Soteropoulos DS, Baker SN (2006) Cortico-cerebellar coherence during a precision grip task in the monkey. J Neurophysiol 95:1194-1206. CrossRef Medline

van Ede F, Jensen O, Maris E (2010) Tactile expectation modulates prestimulus beta-band oscillations in human sensorimotor cortex. Neuroimage 51:867-876. CrossRef Medline

van Ede F, de Lange F, Jensen O, Maris E (2011) Orienting attention to an upcoming tactile event involves a spatially and temporally specific modulation of sensorimotor alpha- and beta-band oscillations. J Neurosci 31:2016-2024. CrossRef Medline

van Ede F, Köster M, Maris E (2012a) Beyond establishing involvement: quantifying the contribution of anticipatory alpha- and beta-band suppression to perceptual improvement with attention. J Neurophysiol 108: 2352-2362. CrossRef Medline

van Ede F, de Lange FP, Maris E (2012b) Attentional cues affect accuracy and reaction time via different cognitive and neural processes. J Neurosci 32:10408-10412. CrossRef Medline

Williams EJ (1959) The comparison of regression variables. J R Stat Soc 21:396-399.

Witham CL, Baker SN (2007) Network oscillations and intrinsic spiking rhythmicity do not covary in monkey sensorimotor areas. J Physiol 580: 801-814. CrossRef Medline

Witham CL, Wang M, Baker SN (2007) Cells in somatosensory areas show synchrony with beta oscillations in monkey motor cortex. Eur J Neurosci 26:2677-2686. CrossRef Medline 